\title{
Climate: patterns, changes, and impacts
}

\author{
Bellie Sivakumar - George Christakos
}

Published online: 28 July 2010

(C) Springer-Verlag 2010

Have the temperatures around the world increased during the last century? Have cyclones, floods, and droughts become more frequent and more extreme? Has the Arctic been shrinking? What about Antarctica? Are the sea levels rising around North America? Are the winters becoming shorter and summers becoming longer in Europe? Is deforestation in the Amazon causing a decrease in precipitation in South America and elsewhere? Are we polluting our environment too much? Will Africa be able to produce enough food to feed its people until 2050? Will there be enough water in Asia to satisfy the needs of its citizens and that of its ecosystems for another 100 years? How many more people will die because of malaria, dengue fever, and other water-borne diseases in this century? What is it with the increasing number of bushfires in Australia anyway?

These are just 'the tip of the iceberg' of questions the world is now grappling with in order to further improve, or at least maintain, our lives, water resources, agriculture, environment, ecosystems, health, economy, and overall well-being. Finding answers to these questions is not at all easy, since the underlying causes and their potential effects

\footnotetext{
B. Sivakumar ( $\square)$

School of Civil and Environmental Engineering, The University

of New South Wales, Sydney, NSW 2052, Australia

e-mail: s.bellie@unsw.edu.au

B. Sivakumar

Department of Land, Air and Water Resources,

University of California, Davis, CA 95616, USA

e-mail: sbellie@ucdavis.edu

G. Christakos

Department of Geography, San Diego State University,

San Diego, CA 92182, USA

e-mail: gchrista@mail.sdsu.edu
}

have far too many different facets that are also often entangled in a difficult web of connections. However, one common denominator in all these, one can confidently say, is our 'climate' system.

Although the vital role of climate on life on Earth has been studied for thousands of years, only since the last few decades has the world been showing increasing concerns about what the climate is doing to us and vice versa. Observations of increases (both number and magnitude) of abnormal hydroclimatic events (e.g. cyclones, floods, droughts, sea level rises) and related ones (e.g. landslides, bushfires) around the world have certainly played an important part in this. A fundamental question that is most often asked is: are the recent extreme hydroclimatic events an indication of a significant (artificial) 'climate change' or simply part of the (natural) 'climate cycle?' If it is perceived to be 'climate change,' then naturally the next question to ask is: what are the causes and effects?

Research on these questions seems to have started as early as 1950s-1960s, but picked up steam only in the 1980s. Since then, however, there has been an extraordinarily steep rise in research activities, journal publications, scientific and other meetings, as well as national and international organizations dealing with these topics, especially 'climate change.' Arguably, the most notable among these is the Intergovernmental Panel on Climate Change (IPCC), established in 1988 by the United Nations Environment Programme (UNEP) and the World Meteorological Organization (WMO) to provide the world with a clear scientific view on the current state of climate change and its potential environmental and socio-economic consequences (http://www.ipcc.ch). The IPCC has so far produced, among others: four assessment reports (AR) in 1990, 1995, 2001, 2007; a supplementary report in 1992; and a special report for emissions scenarios (SRES) in 
2000; the Fifth Assessment Report (AR5) is scheduled to be finalized in 2014. The above initiatives and activities have also led to many important international meetings and agreements linked to the UN Framework Convention on Climate Change (UNFCCC), including the Kyoto Protocol in 1997 and the Copenhagen Summit in 2009; the next UN Climate Change Conference will be held later this year in Cancún, Mexico. Although debates persist on the occurrence of climate change and on the reasons, the general consensus seems to be that climate change is happening and that human activities, especially through emission of greenhouse gases, significantly contribute(d) to it.

This special issue is intended to bring together details of some of the latest climate research activities happening around the world. The Issue consists of 13 papers, contributed by a total of about 40 authors. The papers together cover a whole range of topics in climate research, and may broadly be grouped under three areas: climate patterns, climate changes, and their impacts (especially on water resources). They analyze several hydroclimatic variables (e.g. temperature, humidity, wind speed, solar radiation, precipitation, streamflow), address various water, environmental, and health problems as a result of climate and its change (e.g. droughts, floods, water resources planning and management, thermal properties of lakes, sea levels, dengue fever), apply a host of methods for pattern identification, simulation, prediction, and downscaling of hydroclimatic variables (e.g. statistical methods, nonlinear dynamic techniques, power laws, wavelet transforms, Bayesian methods), and study different regions/countries around the world (Australia, Brazil, China, India, New Zealand, South Africa, South Korea, Taiwan, USA). Also addresssed are the broader issues and challenges in the assessment of impacts of climate change on water resources, including biophysical science challenges, human science challenges, and assessment of water vulnerability.

Acknowledgments The idea for this Special Issue initially came about as a result of the European Geosciences Union (EGU 2008) General Assembly scientific session entitled "Climate, Water and Health." One of us (George Christakos) was the Convener for this session, while the other (Bellie Sivakumar) delivered an Invited Talk. We thank Andras Bardossy, Alin-Andrei Carsteanu, Demetris Koutsoyiannis, and Simon-Michael Papalexiou, who were the co-conveners for the above session and significantly contributed to its success. We very much appreciate and thank all the authors of this Special Issue for their interest and willingness to contribute and also for their hardwork and completion of papers in a timely manner. All the papers submitted to this Special Issue were subjected to a peerreview process, and our sincere thanks to the following reviewers (in alphabetical order) for their timely and constructive reviews: $\mathrm{H}$. Aksoy, V. Barros, R. Berndtsson, J. Chen, Y. Dibike, S. W. Franks, S. Ghosh, J. Griffiths, K. Jinno, L. B. Johnson, J. G. Lay, R. Mehrotra, A. K. Mishra, J. Obeysekera, J. J. O'Brien, B. Rajagopalan, G. B. Sahoo, D. E. Schindler, M. Serre, A. Sharma, S. Srikanthan, C. B. Uvo, V. Venugopal, and A. W. Wood. 